\title{
LA VIOLENCIA POLÍTICA ESTATAL CONTRA EL PUEBLO-NACIÓN MAPUCHE DURANTE LA CONQUISTA TARDÍA DE LA ARAUCANÍA Y EL PROCESO DE RADICACIÓN (CHILE, 1850-1929)*
}

\author{
THE STATE POLITICAL VIOLENCE AGAINST THE MAPUCHE PEOPLE-NATION \\ DURING THE LATE CONQUEST OF ARAUCANIA AND THE SETTLEMENT \\ PROCESS (CHILE, 1850-1929)
}

\author{
Jorge Iván Vergara ${ }^{* *}$ y Héctor Mellado ${ }^{* * *}$
}

\begin{abstract}
En el artículo se realiza una comparación entre la violencia política estatal ejercida por el Estado contra los mapuches de La Araucanía en el período de la conquista (1850-1929) y en el de la entrega de los Títulos de Merced o Radicación (1884-1929). Se identifican y comparan los tipos y formas de violencia así como los agentes estatales o paraestatales responsables. De acuerdo con ello, se concluye que la violencia antimapuche no desapareció con la ocupación del territorio, sino que se prolongó en modos y grados diversos.

Palabras claves: Pueblo mapuche, Nación mapuche, Araucanía, violencia estatal.
\end{abstract}

In this article, a comparison is performed between political violence exercised by the Chilean state against the Mapuche people of Araucanía in the occupation Period (1850-1929) and during the Establishment of the Títulos de Merced o radicación (1884-1929). Different forms of violence are identified and compared, as well as the responsible: state or parastatal. According to this, it can be concluded that the anti-mapuche violence was not eliminated with the occupation of the territory, but continued in different modes and degrees.

Key words: Mapuche people, Mapuche Nation, Araucanía, state violence.

\section{Introducción}

El objetivo de este trabajo es ofrecer una perspectiva histórica y comparativa acerca de la violencia ejercida por el Estado y agentes paraestatales sobre los mapuches de La Araucanía ${ }^{1}$, el principal pueblo indígena del país. Se describirá y comparará dos períodos especialmente importantes: 1) el de la conquista tardía del territorio mapuche, que corre entre 1850 y 1883 y 2) el de la radicación, que abarca entre 1883 y 1929.

Difícilmente se podría poner en duda la magnitud de la violencia en la relación entre el Estado de Chile y el Pueblo-Nación Mapuche, tanto ayer como hoy. No obstante, al querer dar cuenta de este fenómeno nos encontramos con una paradoja en los estudios que se refieren al tema; a saber, que la sola omnipresencia de la violencia dificulta comprender su especificidad. En efecto, la violencia aparece mencionada recurrentemente, pero no elaborada conceptualmente como problema. Ello impide entender, además, sus relaciones con otras formas de dominación o acuerdos políticos no violentos en el marco de los procesos históricos dados. En este sentido, consideramos que la violencia es un instrumento muy frecuente del poder, pero no constituye su fundamento, como se sostiene habitualmente (Arendt, 1969-1970: 138-158).

Se pueden contrastar al respecto las interpretaciones de los historiadores Sergio Villalobos y Leonardo León Solís. De acuerdo con el primero, la ocupación definitiva de La Araucanía no supuso grandes choques militares, y, por esta razón, la violencia no fue un factor de importancia en la "integración" del territorio mapuche:

El avance militar que, en un comienzo, por la falsa imagen de una lucha sangrienta y secular, se pensó sería terrible. Los hechos, fueron, sin embargo, diferentes.

\footnotetext{
* Resultado Proyecto Fondecyt 1141077.

** Universidad de Concepción. Concepción, Chile. Correo electrónico: jorgeivergara@udec.cl.

*** Ayudante Proyecto FONDECYT 1141077. Concepción, Chile. Correo electrónico: hector.mellado.zuniga@gmail.com
} 
No hubo ninguna rebelión formidable y todo se resolvió en palabras altisonantes, parlamentos bien intencionados, temores, amenazas y escaramuzas... La convivencia fronteriza había sido el verdadero factor de una integración iniciada en el siglo XVII (Villalobos, 1982: 64)².

Posteriormente el autor matizó su posición, señalando que: "si bien el alzamiento de 1880-1881 fue duro en algunos momentos, no hubo una rebelión formidable, sino mucho de palabras altisonantes, parlas, temores y amenazas" (Villalobos, 1995: 206)

La interpretación de León Solís subraya, en cambio, la gravitación de la violencia en las relaciones fronterizas, entre ellas en las relaciones entre los propios mapuches, para estos "el límite entre la violencia y la política era muy tenue, especialmente cuando cada segmento pretendía capturar el poder para sobrevivir" (León Solís, 1999: 236), llegando incluso a sostener la existencia del estado de guerra hobbesiano: "A partir de fines de 1772, el mal crónico de la sociedad tribal, la incesante guerra del 'hombre contra el hombre', que describió Hobbes en el Leviathan ${ }^{3}$, floreció con energía en los rehues al sur del Biobío" (León Solís, 1999: 31) ${ }^{4}$. Dicha situación era producto del "quiebre del antiguo consenso, que hizo posible el gobierno colonial" (León Solís, 1999: 34). Un siglo después -concluida la conquista de La Araucanía por el ejército chileno-, "la región quedó sumergida en el caos y la anarquía" (León Solís, 2005: 23), al desmantelarse "los diferentes mecanismos de gobernabilidad que habían hecho posible la compleja vida fronteriza" (León Solís, 2005: 23). Siguiendo a este autor, se comprende que hubo un orden en la Región -anteriormente negado ${ }^{5}$-, pero que fue "sacudido por una brutal guerra étnica", donde se desplegó sin ataduras el "modo de ser fronterizo o afuerino", volviéndose a "la guerra de todos contra todos que caracterizó a la frontera tantas veces en el pasado" (León Solís, 2003: 189 y 268).

Como se puede ver, ninguno de los enfoques proporciona una interpretación válida al problema de la violencia en los dos períodos considerados. Villalobos minimiza la importancia de la violencia política, viéndola solo como un fenómeno secundario, que concluye con la invasión de La Araucanía. León Solís, por otro lado, tiende a exacerbar la violencia como un fenómeno omnipresente y sostenido por todas las partes. Se niega por tanto el papel de los agentes e instituciones estatales en su ejercicio y se sostiene además, contradictoriamente, su disminución e incremento simultáneos.

Desde nuestro punto de vista, el problema de la historia de la violencia política antimapuche no se reduce a la cuestión del mayor o menor grado en que está presente. De igual importancia es entender la diversidad de formas que va adoptando a lo largo del tiempo. Siguiendo a García (1983), hablaremos de "forma" en el sentido de Marx. La "forma" en el pensador alemán significa un modo determinado de constituirse las relaciones sociales, la manifestación de un contenido común, pero expresado de modos diversos y relacionados internamente. Así, la violencia se puede entender como una relación social sustentada en el ejercicio de la fuerza, coerción física, material o amenaza en el uso de dicha fuerza física o coerción (Keane, 1996: 65-70). Cuanto más desnuda sea la presencia de la fuerza, de manera de volverse crecientemente opresiva, menos posibilidad tiene esta de constituirse como relación basada en el consenso o en el convencimiento. De acuerdo con lo señalado acertadamente por Theodor Adorno y Max Horkheimer: "cuando rigen relaciones de poder simples e inmediatas, no existen ideologías en sentido estricto" (1956: 191); esto es, ideas universalizables, capaces de legitimar las relaciones sociales.

Otra limitación inherente a la violencia política es que en un grado extremo conlleva la aniquilación física del adversario. Así, llevada a su plenitud, la puesta en práctica de la violencia podría significar el exterminio mutuo, y, por esta razón, la destrucción de la condición misma de la política: la existencia de los seres humanos (Hinkelammert, 1987). Es posible agregar que individual o masivamente la violencia extiende su efecto más allá de sus víctimas directas, generando pánico y terror entre quienes se busca oprimir.

La violencia -en este caso, la ejercida por el Estado o agentes paraestatales- se podría clasificar en tres tipos principales: 1) el asesinato individual o masivo hasta llegar al genocidio; 2) el daño infligido al cuerpo sin pérdida de la vida humana (las más diversas formas de tortura), y 3 ) las acciones que ponen en peligro las condiciones de reproducción material del individuo o a un grupo, como el despojo territorial, de bienes agrícolas o de otros recursos materiales (casas, herramientas, dinero, etc.). Lo común a estas tres formas -así como a otras cuyo detalle no concierne aquí- es que todas amenazan 
la posibilidad de la vida humana. Por supuesto, que en una misma situación pueden darse varias de estas formas simultáneamente, del mismo modo que un mismo individuo o grupo puede verse afectado por uno o más tipos de violencia a lo largo del tiempo.

\section{La violencia política en la conquista tardía de La Araucanía (1859-1883)}

El pacto colonial suscrito entre los mapuches de La Araucanía y el imperio español estableció como límite entre ambas naciones el río Biobío, reconociéndose un territorio independiente políticamente hasta el Toltén ${ }^{6}$. El Parlamento de Tapihue (1825), ya durante la República, ratificó la autonomía mapuche. Sin embargo, a partir de la década de 1840 el Estado chileno comenzó a cuestionar progresivamente dicho pacto, asumiendo la necesidad de consolidar su presencia en todo el país, frente a otras naciones latinoamericanas y ante potencias europeas.

Los sucesivos gobiernos comenzaron a idear los medios para ocupar La Araucanía, lo que fructificó en diversos proyectos y planes, decidiéndose finalmente por la estrategia elaborada por el coronel Cornelio Saavedra, en 1861; que contemplaba el avance de la línea de frontera hasta el río Malleco, por vía de la construcción de fuertes con el previo pago por el terreno y el emplazamiento de población chilena entre el Biobío y el Malleco hasta controlar todo el territorio (Bengoa, 1985; Pinto, 2002).

Desde los inicios de la ocupación continuó la práctica de parlamentar -con el apoyo de los padres franciscanos establecidos en la región- para acordar la protección del ejército chileno contra enemigos extranjeros, la fundación de fuertes y el asentamiento de no mapuches dentro de territorios ancestrales, invocando el respeto a la autonomía indígena (Saavedra, 1870 y Foerster y Milos, 2005). El proceso experimentó quiebres de importancia cuando, tanto los sectores mapuches aliados del gobierno -principalmente, nagche $^{7}$ y algunos rewe lafkenche $e^{8}$ - como los opuestos a la ocupación de La Araucanía-wenteche $e^{9}$, pewenche ${ }^{10}$ y lafkenchepudieron constatar que las verdaderas intenciones del Estado eran muy diferentes a las declaradas. Pues se buscaba la invasión de la mayor parte del territorio, poniéndolo a disposición de colonos extranjeros y nacionales.

Un aspecto importante dentro del proceso de resistencia mapuche fue el pensamiento y acción de uno de los líderes wenteche de la época, Magiñ Wenu. El gran lonko buscó reaccionar frente al inminente avance de la frontera y la fragmentación del territorio a partir de la compra para la creación de fuertes y población chilena en el Wallmapu -justificada por la protección de los especuladores y usurpadores de sus tierras que se venían estableciendo desde hace décadas en La Araucanía-. Por medio de misioneros como Palavecino intentó que el Estado dejara la idea de ocupar el territorio a partir de los tratados anteriormente celebrados (Marimán et al, 2006: 103).

Desde el punto de vista del ejercicio de la violencia estatal como método de sometimiento, podemos identificar dos momentos claramente diferenciados: el primer período va desde 1861 a 1869, bajo la dirección de Cornelio Saavedra, y el segundo desde 1869 a 1883, bajo el mando de José Manuel Pinto, Basilio Urrutia y Gregorio Urrutia, respectivamente. En el primero, la violencia estuvo más bien localizada y tuvo su origen en problemas relativos a la propiedad de la tierra y a la usura por chilenos y agentes del Estado. En el segundo, se intensificó el uso de la fuerza por parte de las tropas chilenas, afectando sembradíos, viviendas, animales y personas no combatientes.

Si bien las leyes establecieron cierta protección para los mapuches, estas tuvieron en la práctica menos impacto de lo esperado, porque no frenaron el avance de las usurpaciones, algunas de ellas realizadas por métodos violentos. Podemos reconocer la incipiente presencia de la fuerza, principalmente en manos de los colonos espontáneos, cuya penetración en La Araucanía no era nueva, pero que se fue acrecentado con el avance de la ocupación chilena. Recordemos que dicha infiltración había sido el principal motivo del levantamiento de 1859 , sirviendo como el pretexto inmediato para iniciar la conquista del territorio mapuche (Bengoa, 1985: 170; Pinto, 2002: 145) ${ }^{11}$.

Cornelio Saavedra, que conocía claramente las enemistades entre las espacialidades territoriales de Arauco y Malleco, dispuso emisarios con el objetivo de presentar su plan de ocupación a los mapuches, expresando la necesidad de establecer plazas militares para la defensa de chilenos y mapuches frente a los criminales de la frontera que se amparaban entre ellos y les causaban muchos perjuicios. Esta propuesta fue bien acogida por la mayor parte de los grupos lafkenche y nagche ${ }^{12}$.

En nombre del Estado, y conforme se iba produciendo el avance hacia el sur, el coronel Saavedra 
fue comprando tierras a los mapuches y abriendo espacios para fuertes, asentamientos y futuras colonias extranjeras, buscando a la vez terminar con la violencia ejercida por especuladores y estafadores en la compra-venta de terrenos a mapuches ${ }^{13}$. Era la lucha del Estado contra la violencia de los particulares hacia los mapuches ${ }^{14}$. Dichos terrenos fueron siendo medidos y vendidos hasta el fin de la ocupación ${ }^{15}$. De acuerdo con las cifras entregadas por Teodoro Schmidt, presidente de la Comisión de Ingenieros -encargada de hacer las mensuras de los terrenos de La Araucanía-, en 1873, ya se habían medido 123.700 ha, de estas solo 13.073 ha, $10,56 \%$, fueron reconocidas ("entregadas", según Schmidt) como propiedad mapuche. El resto había sido rematado $(37,28 \%)$ o estaba en poder de particulares $(45,66 \%)$ y de colonos nacionales $(6,46 \%)$, una distribución claramente perjudicial para los mapuches ${ }^{16}$.

Los episodios de violencia ocurridos en esta primera fase de la invasión son consecuencia de la usura indiscriminada a fines de la década de 1850 y comienzos de la siguiente; momento en que la legislación chilena recién comenzó a plantear la necesidad de ocupar el Wallmapu. Un importante testimonio al respecto es la carta de Mangil Wenu al presidente del país, Manuel Montt, de septiembre de 1860 , donde señala:

\begin{abstract}
"En noviembre llegó otro intendente que llaman Villalón ${ }^{17}$, y que el día 24 en la noche se apareció en nuestras tierras trayendo mil hombres y mostrando cañones, y se les dejaron caer a los mapuches, bureanos, reinaquinos y murchenos y les robaron todos sus animales, cuyo número se estima en los nueve mil caballos, yeguas, vacas y ganado ovejuno. Hubo quemas de casas y se llevaron cuanto encontraron en ellas, porque alcanzaron las familias que iban a esconderse en los montes. Los indios viejos y las indias que no pudieron llevar las degollaron como perros. El mismo Salbo hizo degollar a un indio" 18 .
\end{abstract}

Al leer este texto se constata que en las usurpaciones, robos y asesinatos existía participación de autoridades provinciales -incluido el propio intendente de la provincia de Arauco- y locales como el capitán de infantería y comandante de armas de Santa Bárbara, Domingo Salvo ${ }^{19}$.
No se trataba solo de disputas en juicios por tierras, el desplazamiento de la frontera profundizaba la lucha y la acción directa contra la sociedad mapuche. Las tropas chilenas comenzaron a atacar rewe aledaños a las fortificaciones, lo que causó un cambio en el ejercicio de la guerra. Los mapuches optaron por no enfrentar directamente al ejército, por ello a fines del mandato de Saavedra, en 1869, se realizó un parlamento para llevar a la paz el territorio.

Al dejar Saavedra el puesto de jefe militar ese mismo año, se dio inicio a la fase más violenta de toda la ocupación. Nos referimos a la resistencia a partir de la rebelión encabezada por el Lonko Kilapán, Nidol Toki ${ }^{20}$ del Weichanmapu ${ }^{21}$, el que enfrentó al ejército de ocupación comandado por los generales Pinto y Urrutia. Entre 1869 y 1881 se produjeron los hechos más significativos de violencia estatal contra mapuches durante el conflicto, con el nuevo avance de la frontera hacia los ríos Traiguén y Cautín, respectivamente. Un testimonio temprano lo constituye la comunicación del intendente de Valdivia, Epifanio del Canto, al ministro de Guerra, en mayo de 1871, donde subraya los peligros que suponían para la provincia:

"La pobreza a que están reducidos los indios araucanos ${ }^{22}$ que residen al sur del río Cautín, como consecuencia de la constante persecución que se les hace de la parte del norte por el ejército de la línea del Malleco", agregando que esto ha sido la causa de que "muchos de ellos han venido a ponerse en contacto con los indios que habitan dentro del territorio de esta provincia"23.

Tal proceso se agudizó en los años siguientes. Como señala el general Basilio Urrutia en 1876:

Sabemos perfectamente que las tribus arribanas han abandonado por completo sus dominios aquende el Cautín, replegándose al sur de ese río y a los últimos rincones de la cordillera de los Andes. La región subandina se halla, pues, vacante y completamente deshabitada" (Urrutia, 1876: 7).

Uno de los primeros hechos del que podemos dar constancia ocurrió el 12 de enero de 1869, cuando una persona de nombre Enrique Greene pidió 
permiso al general José Manuel Pinto para reunir un grupo con el fin de robarle animales a los pewenche de la cordillera. La expedición, consistente en 52 hombres, a cargo de un tal Saldías, tenía la orden de "robar cuanto pudieren, y matar a chico y grande, fuesen hombres o mujeres" 24 . Es así como atacó un pequeño rewe en Lolco, un territorio pacífico. Saldías volvió con una gran cantidad de animales y después de haber asesinado a 20 pewenches, entre hombres, mujeres y niños, y cautivado 3 niños (Bengoa, 1985: 218). Este episodio es relevante puesto que es el único donde vemos que fuerzas no militares realizan una masacre de tal magnitud.

Un año después, el 28 de enero de 1870 -relata Robustiniano Vera- una compañía de Granaderos encontró a un grupo de mapuches robando ganado. Los atacantes habrían dado muerte a un soldado y a dos colonos, por lo que se les persiguió por una legua. La situación derivó en una lucha de arma blanca con 11 mapuches muertos y el botín recuperado (Vera, 1905: 73). Se trató ciertamente de una matanza, pues la unidad militar no respetó las prescripciones oficiales, que indicaban que debían haberse tomado prisioneros para la realización de un juicio por los delitos cometidos y no matarlos en el acto $^{25}$.

A inicios de la década siguiente los hechos de violencia estatal contra los mapuches se multiplicaron, produciéndose graves y reiterados incidentes. Veamos algunos de los más importantes. De acuerdo con Tomás Guevara, el 7 de mayo de 1880 en las cercanías de Chanco una división de 440 hombres se dirigió hacia los asentamientos mapuches. Al llegar a la ribera del río Dumo se adelantaron 240 hombres al mando de Bonifacio Burgos, asaltando a los rewe de Pailahueque, Nanqucheo y Pancho, donde asesinaron a 13 personas y tomaron 15 prisioneros y gran cantidad de animales (Guevara, 1902: 369).

El 10 de noviembre de 1881 tuvo lugar lo que Gavilán denomina "la masacre de Temuco", donde 3.500 mapuches asentados en la futura ciudad y sus alrededores fueron sorprendidos por fuerzas del Ejército, quienes los asesinaron, causando un impacto profundo en la sociedad mapuche por la crueldad del acto, en lo que hasta hoy se conoce como el sector de Matanza (Gavilán, 2007: 114-115).

Un hecho similar ocurrió en Lumaco solo dos días después, el 12 de noviembre de 1881, cuando fueron asesinados 11 mapuches dentro de las celdas del fuerte en extrañas circunstancias. Es posible profundizar en este episodio, porque se cuenta con una documentación mucho más completa que en ningún otro caso gracias al sumario realizado por el mismo Ejército $^{26}$. Los hechos se iniciaron cuando un soldado fue a dormir junto con los prisioneros dentro del primer calabozo que daba hacia la calle, y sin que lo supiesen los demás soldados del cuartel. Durante la noche los mapuches le habrían propinado un palo en la cabeza, dándole muerte al instante. $\mathrm{Al}$ darse cuenta el resto de la tropa y los oficiales, se ordenó el ingreso de soldados a las celdas, siendo fusilados tres de los cinco mapuches, quienes se defendían con palos de leña y barras de la celda. Al verificar si todos estaban muertos, se encontraron con dos mapuches vivos, los que fueron asesinados con sus espadas por los oficiales Roselot y Casanueva.

Unas horas más tarde se produjo un segundo hecho de sangre. Según una primera versión de parte de los soldados, siendo casi de madrugada se dio la alarma, pues los otros cinco presos mapuches se habrían alzado en la celda contigua. Todos ellos fueron fusilados por la guardia del cuartel, sumando once muertos. Contradiciendo esto, el fiscal Ezequiel Villarroel, según su investigación, señaló que estaban en una actitud de perdón y tirados en el suelo. Más aún, el fiscal también puso en evidencia las incongruencias entre las declaraciones de los militares, dejando sin efecto el primer sumario e iniciando otro. Pese a ello, el proceso terminó aparentemente sin culpables.

Otro suceso importante ocurrió el 13 de diciembre de 1882 en Alto Biobío. Por orden de los mayordomos de la Hacienda San Ignacio, un grupo de militares mató a diecinueve personas -entre mujeres y niños- y quemaron sus cuerpos. Posteriormente asesinaron a diez hombres que estaban bajo el mando del cacique de Lonquimay. El sumario realizado para esclarecer los hechos reveló que habían dado muerte a otros siete mapuches antes, lanzándolos al río para esconder el crimen, contabilizando treinta y seis muertos (Guevara, 1902: 469-470).

Según lo visto, la segunda parte del proceso de ocupación de La Araucanía se caracterizó por la presencia de formas de violencia propias de una guerra sucia, en la que el asesinato de personas inocentes o apresadas sin resistencia se volvió un hecho común. Un punto de relevancia es la falta de enjuiciamiento a los responsables en todos los casos relatados; aún más, en Lumaco y Alto Biobío donde sí hubo juicios, no arrojaron culpables. Al 
comparar ambas fases se puede concluir que, bajo la comandancia de Saavedra, no se registró ningún hecho de violencia de la magnitud o similar a los realizados durante las comandancias de Pinto y de los Urrutia. Los hechos ocurridos durante su comandancia estuvieron relacionados con problemas de propiedad de la tierra, dados entre mapuches y colonos por los límites que involucraban usura o estafa, pero siempre en el plano local. En cambio, en la segunda fase encontramos una violencia más generalizada por parte de los militares: la matanza de prisioneros, de rewe y de grupos de mapuches sin mediar enfrentamiento previo, además de la práctica sistemática de destrucción de casas y cultivos, de robo de animales y aun de asesinatos de niños.

\section{La violencia bajo la radicación (1884-1929)}

El proceso de radicación no significó el fin de la violencia política estatal contra los mapuches. Más aún, adquirió nuevos contornos con la entrada de colonos chilenos y extranjeros, muchas veces apoyados por la policía (Carabineros o Gendarmes de la Colonia). Principalmente se acrecentaron las acciones de fuerza destinadas a apropiarse de terrenos mapuches. El presidente de la Sociedad Caupolicán Defensora de La Araucanía, Manuel Antonio Neculmán, señala al respecto:

El procedimiento de que se valen los particulares para apoderarse de los intereses de los indígenas es lo más ilegal y condenado por las leyes de nuestro país. El indígena no tiene ninguna garantía en su hogar ni en su vida, en todo momento se ve expuesto a ser arrebatado de su domicilio que es inviolable, según la Constitución Política del Estado, por hechos que muchas veces pueden ser fingidos y que solo tienen como objeto apoderarse de los intereses de los indígenas, cuyos hechos son numerosos"27.

Neculmán denuncia al intendente de Cautín lo ocurrido con dos mapuches del sector de Quepe, Yane Filuche y su sobrino Curiqueo, a cuya vivienda había llegado un grupo encabezado por un agricultor, José del Carmen Sepúlveda, más otra persona y tres carabineros. Estos últimos habían procedido a amarrarlos y azotarlos, para después llevarse a Curiqueo al domicilio de Sepúlveda. Allí lo mantuvieron "colgado en un roble, atormentándosele a golpes y que declarase quién era el autor del robo de un animal que decía Sepúlveda le había sido hurtado y como dijera que nada sabía fue sumergido en el estero donde se le sacó sin sentido" 28 . Mientras Filuche fue dejado libre al día siguiente por intermediación de un tercer mapuche, Curiqueo fue llevado al fundo de Nicanor Hidalgo, "donde permaneció detenido por cuatro días, haciéndosele alojar en un cepo sin tener con qué cubrirse, sin atender la inclemencia del tiempo, ni su ancianidad, (setenta años), y que por fin se le dejó en libertad el domingo del presente"29. Neculmán agrega que:

Estos hechos contra los indígenas, señor Intendente, son frecuentes, basta con que cualquier particular se presente denunciando un robo o hurto para que se arranque de su hogar al primer indígena que aquel indique y que siempre tiene que ser uno que tenga a lo menos una yunta de bueyes y someterlo a los más crueles tormentos hasta arrancarle un convenio en que se declare culpable ${ }^{30}$.

El capitán a cargo del Escuadrón de Carabineros desmiente la denuncia anterior, tildándola de "antojadiza y falaz". Adjunta el informe del juez de letras, quien también descalifica las acusaciones de tortura como "una imputación tan antojadiza como falsa y que solo existe en la mente de malos individuos para desprestigiar a los carabineros, inducidos a esto por tinterillos de mala lengua que explotan a la gente ignorante haciéndola incurrir en falsedades para lucrar con ellos"31. Como queda en evidencia, existía una complicidad abierta entre la policía, los colonos y la justicia, que impedía poner fin a los múltiples y reiterados actos de violencia contra los mapuches, generalmente para despojarlos de sus bienes y tierras.

También se pone de manifiesto que, consumada la derrota militar y política mapuche, la disputa por tierras había adquirido una nueva dimensión. En efecto, las dos principales formas de violencia relacionadas con ellas en el período de la radicación fueron: 1) el despojo legal-realizado por el Estado por medio de sus agentes civiles y militares- y 2) el despojo ilegal, llevado a cabo principalmente por colonos chilenos y extranjeros recién establecidos en los lindes de los nuevos terrenos mapuches formados por los Títulos de Merced y que abarcaban una muy pequeña extensión de su antiguo territorio (6\% aprox.). 
Debe recordarse siempre que la radicación no podía tener efecto si los mapuches no se encontraban en posesión de los terrenos, por lo que las tierras que fueron adquiridas o expoliadas a ellos con anterioridad no podían ser objeto de la acción de la Comisión Radicadora. El presidente de la Comisión Radicadora, Leoncio Rivera, en su Informe Anual correspondiente al año 1911-1912, explica que:

\begin{abstract}
El servicio en la parte austral del territorio indígena va siendo cada día más engorroso a causa de los diversos sistemas puestos en práctica para el acaparamiento de las tierras de indígenas; i me cabe repetir hoy, que si no se dictan disposiciones especiales, protectoras de la propiedad, o de mayor eficacia para la labor de los Protectores de Indígenas, encontraremos cada día mayores dificultades, i concluiremos por quedar del todo paralizados por falta de campo para ejercitar nuestra acción: pues, ya casi no hay terreno pretendido por indígenas que no se halle en discusión con particulares, con derechos más o menos acentuados de posesión o dominio. Para radicar en condiciones de servicio expedito i eficaz, es preciso primero reivindicar" 32 (destacado míos)
\end{abstract}

En efecto, los principales problemas de la nueva configuración de La Araucanía guardaban relación con demandas por parte de gente mapuche al Estado o personas de relevancia político-nacional respecto de despojos de tierras y violencia, muy vinculadas entre sí. Por ejemplo, en 1891 el lonko Pedro Kayupi se dirigió al superior de la Orden Franciscana para que intercediera en el inminente remate de las tierras de las comunidades de Coyinco y Chanco, que comprendían 150 familias en el departamento de Nueva Imperial. Este solicitó que, además de cancelar los remates, se le otorgase un título de dominio por las tierras (Pavéz, 2008: 794-795).

$\mathrm{Al}$ despojo de tierras de parte del Estado y de los colonos se añadió luego un nuevo elemento: el uso de la fuerza pública para defender las tierras ya usurpadas y de esa manera controlar todo el territorio ocupado. En noviembre de 1896, a nombre de su familia y de "muchos y prestigiosos caciques de La Araucanía", Esteban Romero escribió una carta al Presidente de la época, Federico Errázuriz, donde relata el despojo sufrido por él y numerosas familias que pertenecieron a su reducción. En un pasaje significativo señala que:

Las autoridades en representación del Estado i a nombre de la Ley, cooperan al despojo que nos hacen los especuladores de tierras y animales en la frontera, obligándonos a abandonar lo que tanto amamos i en que hemos vivido con nuestros padres, en la que sus restos descansan, con la que hemos alimentado a nuestros hijos i regado nuestra sangre...A nosotros se nos martiriza i trata de esterminar, de todos modos. Las policías rurales nos vejan i quitan nuestros caballos $i$ se nos hace responsables de cualquier robo que en la frontera se ejecute; se nos arrastra a la cárcel i allí se nos maltrata cruelmente i tenemos que sufrir el hambre i morir, de pena i est[enuacion]"33.

Un caso igualmente dramático al respecto fue el de la comunidad Juan Pinchuleo, de Quepe, en abril de 1913. Dicha comunidad pidió y obtuvo del Estado las hijuelas 154, 155, 156 y 157, pero el agricultor Francisco Tejeda compró también tres de las cuatro hijuelas $(155,156$ y 157) a Carlos Concha Subercaseaux, avecindado en Santiago, en la suma de \$ 5.500. El problema llegó a Tribunales y durante largo tiempo no hubo sentencia, ya que en el momento en que las autoridades quisieron levantar los planos de las tierras en litigio, unos 60 comuneros resistieron las operaciones. El 17 de marzo de 1917 fueron expulsados por carabineros con una violencia desmedida, particularmente hacia las mujeres de la comunidad (Pinto, 2015a: 60-62). En una presentación hecha por la Sociedad Caupolicán al Inspector General de Colonización y Emigración se responsabiliza a la Comisión Radicadora de Indígenas, que había declarado fiscales los terrenos, haciendo prevalecer sus intereses "sobre los más justos derechos de nuestra raza" 34 .

Por otro lado, desde inicios del siglo XX los mapuches comenzaron a reorganizarse para idear nuevos mecanismos de lucha y revitalizar los antiguos, visibilizando y enfrentando la violencia ejercida en su contra. El hito más significativo y presente en la memoria mapuche del presente ha sido el parlamento de Coz-Coz, Panguipulli, en 1907, que tuvo dos peculiaridades. La primera es que se recurrió a una institución propia del pueblo mapuche para unirse y reflexionar frente a la violencia 
y el despojo que los afectaban y la segunda es que a dicho mecanismo ancestral se sumaron nuevos elementos como fue la presencia de un periodista que plasmó en El Diario Ilustrado todo lo que pudo anotar y recordar (Díaz Mesa, 1907).

De acuerdo con los testimonios mapuches, la costumbre de utilizar un notario para manipular contratos siguió durante el proceso de radicación, pues así se les engañaba para apropiarse de una parte importante o de la totalidad de la reducción. El robo de animales fue también frecuente durante la ocupación y en el período de radicación se profundizó aún más. Las compañías ganaderas y los nuevos dueños del territorio crearon cuadrillas especializadas para el robo de animales. El mecanismo era simple: llegar a un territorio mapuche y robar durante la noche o esperando que nadie se encontrase, de no ser así se intimidaba y violentaba a los moradores. Otro modo usual era emborracharlos y entregarles migajas por sus animales y terrenos, dejándolos en la indefensión. Asimismo, era muy común incriminarlos falsamente de acciones de robo, para luego tomar acciones en su contra: azotes, detenciones, rapto con violencia y tortura para lograr la venta o la huida a los territorios colindantes (Díaz Mesa, 1907: 238-242)35.

Otro mecanismo aún más violento fueron los asesinatos. En el Parlamento se refirieron los casos de Nieves Ayñamco, mandada a asesinar por Joaquín Mera $^{36}$ y de la Compañía San Martín, empresa de colonización que se adueñó del lago Panguipulli, destruyendo toda embarcación mapuche que circulara por él y responsable también de los asesinatos de Mariano Millanguir y de su hijo Manuel, por los que no respondieron ante la justicia (Díaz Mesa, 1907: 246-252; Fraunhäusl, 1904-1924: 326-327). Solo unos meses después de realizada la junta en Coz-Coz, tuvo lugar en Panguipulli otro nuevo asesinato contra un mapuche. Así es como el padre Sigisfredo estampó en su diario el acontecimiento:

"El 24 de junio fue encontrado en el estero de Coz-Coz el cadáver del anciano indígena Neculpán. Según las demostraciones del cadáver, el indio había sido estrangulado y después echado al estero.

"Al principio se creyó que el hechor del crimen sería José Calipán. Unos 10 indios lo trajeron a la misión. Interrogado por mí declaró que no era él quien había cometido el crimen sino Rosario Sanhueza, inquilino de Joaquín Mera, y Adolfo Jaramillo declaró haber visto salir de la tomadura de Isidoro Jaramillo a Neculpán, haber presenciado que Sanhueza y Jaramillo le seguían, lo echaban al suelo, lo estrangulaban y lo botaban al estero.

"Los [mal]hechores fueron llevados a Valdivia y estuvieron 6 meses en la cárcel. Como los dolientes no merecieron mejorarse de pruebas, Sanhueza y Jaramillo fueron absueltos y puestos en libertad.

"Joaquín Mera se encargó de la casa y del terreno del indio. La casa la quemó y el terreno lo cercó" (Fraunhäusl, 19041924: 330)

Además de la impunidad en que quedó el crimen, llama la atención que uno de sus instigadores o, al menos, cómplice, se apoderase de las tierras del asesinado.

Por otro lado, en muchos casos, la misma policía aparecía involucrada, como lo ilustra la denuncia hecha en julio de 1912 por Manuel Antonio Neculmán, presidente de la Sociedad Caupolicán, al intendente de la provincia de Cautín, donde solicita investigar la muerte de Juan Marileo en un cuartel de Chol-Chol, "causada por los golpes y heridas recibidas de los carabineros" y luego de ser traído con un lazo al cuello ${ }^{37}$. El juez de subdelegación respectivo señala que no le corresponde investigar su muerte, porque Marileo no presentaba lesiones y que, además, según los testimonios de sus compañeros de celda, había muerto de frío a causa de la falta de cobijas.

Además de los señalados, existieron también otros tipos de violencia que no tenían necesariamente que ver con la disputa de tierras o el despojo y que dan cuenta de que la violencia comprendió todos los ámbitos de la vida (Cf. León-Solís, 2003 y 2005 y Pinto, 2015a y 2015d). En efecto, el establecimiento de las nuevas urbes en la frontera dio lugar a numerosos abusos. José Miguel Varela, relata en su autobiografía lo insegura que era la ciudad de Temuco debido a los bandidos que asechaban constantemente a chilenos y mapuches:

"Cuando los mapuches no tenían dinero para comprar alcohol, nunca faltaba el sinvergüenza que les pasaba unas pocas monedas a cambio de animales, tejidos, provisiones y hasta sus tierras, para ello contaban con los solícitos servicios de un 
tránsfuga que oficiaba como notario, que atendía las veinticuatro horas del día, de lunes a domingo" (Varela, 2015: 313)

Otro hecho muy emblemático ocurrió en 1913, la denominada "marcación Painemal", un evento repudiado masivamente por la comunidad mapuche por el grado de violencia que revistió. El acto de marcar a un mapuche por parte de un colono que lo acusaba sin evidencia de un robo, reveló la profunda desigualdad e injusticia presente entre colonos europeos y mapuches. La repercusión fue tal que la Sociedad Caupolicán convocó a una manifestación en Nueva Imperial, a la que asistieron más de tres mil mapuches para repudiar el hecho y dejar en claro que no se podía dejar en la impunidad ningún otro acto de violencia contra su pueblo ${ }^{38}$.

\section{Conclusiones}

Descritos algunos de los hechos más relevantes de violencia política estatal en cada período -invasión y radicación, respectivamente-, buscaremos hacer una comparación entre ellos, de manera que sea posible dar cuenta tanto de los aspectos comunes como de las diferencias. De acuerdo con lo visto, en ambos periodos la violencia jugó un papel muy importante, incluso central, en debilitar la resistencia mapuche. Podemos destacar cuatro aspectos relacionados con la violencia en ambos procesos: los sujetos históricos, el tipo de violencia, el rol de Estado y, secundariamente, las acciones de defensa mapuches.

Los sujetos históricos que ejercen la violencia cambian en cada período. En el proceso de invasión, en primer lugar, fueron responsables las Fuerzas Armadas -incluidos el ejército y la armada-, en segundo lugar, la Guardia Cívica. Estos agentes llevaron a cabo la ocupación de La Araucanía y realizaron los principales actos de violencia. Los soldados victoriosos de la Guerra del Pacífico en contra de Perú y Bolivia realizaron el último avance de Temuco a Villarrica, esperanzados en obtener alguna ganancia de dicha empresa, de la que finalmente no obtuvieron nada. Decepcionados del gobierno chileno, vieron una solución a su pobreza obteniendo trozos de tierra y bienes del territorio mapuche. Durante el proceso de radicación, adquirió gran relevancia la figura de la policía, cuya tarea era el resguardo de las nuevas poblaciones, pero que cometió numerosos abusos contra los mapuches. Sin embargo, fueron los colonos extranjeros y nacionales quienes asumieron el protagonismo de la violencia antiindígena, del asesinato de personas mapuches y del robo de sus tierras, bienes y animales. La negligencia -cuando no la complicidad de las instituciones estatales-, en la administración de la justicia y garantizar la seguridad de la población, permitieron la ocurrencia y reiteración de estas situaciones.

En cuanto al tipo de violencia, también se pueden constatar importantes diferencias. Durante la conquista de La Araucanía, el ejército perpetró acciones violentas para debilitar a la población mapuche que, en la actualidad, se considerarían graves violaciones a los derechos humanos. Se sucedieron varias matanzas, entre las que podemos señalar las de Lolco (1869), Lumaco (1881) y Alto Biobío (1882). En efecto, a partir de la llegada a la comandancia de José Manuel Pinto (1869) a la frontera, el enfrentamiento se transformó en una guerra sucia, con los soldados chilenos quemando siembras, robando animales, destruyendo casas y provocando terror en la población mapuche, cuya resistencia militar se vio cada vez más disminuida. En cambio, durante el período de la radicación predominó la violencia de los colonos para apoderarse de las tierras y bienes mapuches, recurriendo tanto al engaño como a la fuerza. Lo más recurrente no fueron las matanzas, sino los asesinatos a propietarios mapuches.

El tercer aspecto a considerar dice relación con el rol del Estado en ambos procesos. Respecto de la conquista tardía de La Araucanía, dicho papel en la generación de la violencia fue muy activo. Por medio de sus leyes estableció las bases para dar aparente legalidad a la invasión, la que fue emprendida no solo con negociaciones y diplomacia -sobre todo durante el mando de Cornelio Saavedra-, sino también por la vía militar, muy a menudo sobrepasando la propia legislación vigente, como fue el caso de las masacres antes mencionadas. En el caso de la Radicación, y como hemos señalado, la violencia fue principalmente ejercida por los colonos, que en la medida de que eran objeto privilegiado de la protección estatal, cumplían la función de agentes paraestatales. Por tanto, la ausencia del Estado es aparente, ya que no solo no previó los resultados de una política de colonización extranjera completamente excluyente de los mapuches y aun de muchos chilenos pobres, víctimas también de la violencia ${ }^{39}$, sino que no sancionó los abusos cometidos por parte de los colonos, ni de la fuerza policial. 
El último problema a abordar es la defensa ejercida por los mapuches. Durante el proceso de invasión, iniciado con la expoliación legal e ilegal de la propiedad mapuche en el norte del Ngulumapu, la defensa del territorio se dio originariamente mediante negociaciones y demandas a los jefes militares y autoridades políticas. Con el cambio de mando en la Comandancia del Ejército y la guerra sucia puesta en práctica por las FF.AA., los mapuches se enfocaron en la defensa del territorio que no estaba ocupado para la época (1870 - Malleco). Se realizaron Trawun (juntas) para reorganizar las fuerzas que estaban bajo la frontera. En ellas se dio a conocer el Toki general del ejército mapuche, Kilapán, hijo de Magiñ Wenu, quien ejerció el mando hasta las postrimerías de la conquista de La Araucanía.

A su vez, bajo la radicación, la sociedad mapuche re-articuló sus fuerzas, generando nuevas formas de organización y lucha. Desde 1900 las organizaciones mapuches más importantes de las primeras décadas del siglo XX, la Sociedad Caupolicán y la
Federación Araucana, fueron la mayor expresión de este cambio (Foerster y Montecino, 1988). Asimismo, se establecieron vínculos con la FOCH, Federaciones de Estudiantes y partidos políticos, buscando canalizar u obtener apoyo a sus demandas. Predominaron las alianzas con la izquierda y los sectores populares, pero también hubo relaciones con la Iglesia católica, el Partido Conservador y sectores liberales.

Finalmente, es interesante señalar que, si bien hacia la década de 1930, consumada ya la radicación, los hechos violentos tendieron a disminuir, no se puede desconocer que la violencia estatal y paraestatal se mantuvo latente, para resurgir con fuerza frente a acontecimientos como el levantamiento de Ranquil, en 1934, donde numerosos mapuches y colonos chilenos fueron asesinados; y cobró ribetes altamente represivos después del golpe militar de 1973, renaciendo nuevamente a fines de los años 1990, con la represión policial de las comunidades mapuches más activas políticamente.

\section{Referencias Citadas}

Adorno, Theodor W. y Horkheimer, Max

1956 "La ideología", en: La sociedad. Lecciones de sociología, Ed. Proteo, Buenos Aires, 1969, pp. 183-205.

Ancán, José

2008 "Prólogo. Las múltiples caras del engaño", en: Crónica militar de la conquista y pacificación de la Araucanía desde el año 1859 hasta su completa incorporación al territorio nacional, Navarro, Leandro, Pehuén Editores, Santiago, pp. 15-23.

Anuario Estadístico

1870 Anuario Estadístico de la República de Chile correspondientes a los años de 1868 i 1869, Imprenta Nacional, Santiago.

Arendt, Hanna

1969-1970 "Sobre la violencia", en: Ídem, Crisis de la república, Taurus, Madrid, 1998, pp. 109-200.

Bengoa, José

1985 Historia del pueblo mapuche, Eds. SUR, Santiago, 2da Ed. 1987.

Bengoa, José

2007 El tratado de Quilín, Catalonia, Santiago.

Berger, Luis

2014 "La matanza de colonos nacionales de Loncoche (1910)", en: Revista Austral de Ciencias Sociales 27, pp. 71-102.

Contreras Painemal, Carlos

2010 Los Tratados celebrados por los Mapuches con la Corona

Española, la República de Chile y la República de Argentina, Tesis de Doctorado en Altamerikanistik, Universidad Libre de Berlín (versión disponible en sitio web: http://www. archivochile.com/carril_c/cc2013/cc_2013_00009.pdf, visitado 1.9.2017).

Díaz Meza, Aurelio

1907 "En la Araucanía. Breve relación del último Parlamento araucano de Coz-Coz en 18 de enero de 1907", en: En la Araucanía. El padre Sigifredo de Frauenhäusl y el Parlamento mapuche de Coz-Coz de 1907, Carmen Arellano et al (eds.), Vervuert, Madrid y Fráncfort del Meno, 2006, pp. 195-264.

Foerster, Rolf

1993 Introducción a la religiosidad mapuche, Ed. Universitaria, Santiago, 2da. Ed. 1995.

Foerster, Rolf

1996 "La propiedad huilliche en los Llanos de Valdivia y Río Bueno", en: La propiedad huilliche en la provincia de Valdivia, Vergara, Jorge Iván; Mascareño, Aldo y Foerster, Rolf, CONADI, Santiago, pp. 243-371.

Foerster, Rolf y Milos, Diego

2005 Pacificación de la Araucanía: correspondencia del P. Buenaventura Ortega (segunda parte), Publicaciones del Archivo Franciscano, Santiago.

Fraunhäusl, Sigifredo de P.

1904-1924 "Crónica de la Misión de San Sebastián de Panguipulli”, en: En la Araucanía. El padre Sigifredo de Frauenhäusl y el Parlamento mapuche de Coz-Coz de 1907, Carmen Arellano et al (eds.), Vervuert, Madrid - Fráncfort del Meno, 2006, pp. 297-382.

García, José Fernando

1983 Explicación dialéctica y explicación positivista en ciencias sociales, FLACSO-Chile, Contribuciones $\mathrm{N}^{\circ} 15$, Santiago. 
Gavilán, Víctor

2007 La Nación Mapuche = Puelmapu ka Gulumapu, Ed. Ayún, Santiago.

Guevara, Tomás

1902 Historia de la civilización de la Araucanía. Tomo III. Los araucanos i la república, Imprenta Barcelona, Santiago.

Hinkelammert, Franz

1987 "El concepto de lo político según Carl Schmitt", en: Ídem, Democracia y totalitarismo, Amerindia, Santiago, pp. 211-228.

Hobbes, Thomas

1651 Leviathan, Oxford University Press, Oxford-Nueva York, 1998.

Keane, John 1996 On violence, Polity Press, Londres.

León Solís, Leonardo

1990 Maloqueros y conchavadores en Araucanía y las Pampas, 1700-1800, Ediciones Universidad de La Frontera, Serie Quinto Centenario, Temuco.

León Solís, Leonardo 1992 "El pacto colonial hispano-araucano y el parlamento de 1692", en: Nütram VIII (30), Santiago, pp. 27-53.

León-Solís, Leonardo 1993 El parlamento de Tapihue, Eds. Rehue, Santiago.

León Solís, Leonardo 1999 Apogeo y ocaso del Toqui Ayllapangui de Malleco, Chile. 1769-1776, DIBAM, Santiago.

León Solís, Leonardo

2003 "Violencia inter-étnica en la Araucanía, 1880-1900", en: Araucanía: la frontera mestiza, Siglo XIX, León, Leonardo et al, Ediciones Universidad Católica Cardenal Raúl Silva Henríquez, Santiago, pp. 181-286.

León-Solís, Leonardo

2005 Araucanía: la violencia mestiza y el mito de la "Pacificación”, 1880-1900, Universidad Arcis, Santiago.

Marimán, Pablo

2006 "Los mapuches antes de la conquista militar chilenoargentina", en: i...Escucha, Winka...! LOM Ediciones, Santiago, pp. 53-127.

Navarro, Leandro

1909 Crónica militar de la conquista y pacificación de la Araucanía desde el año 1859 hasta su completa incorporación al territorio nacional, Pehuén Editores, Santiago, 2008.

Pavéz, Jorge (Comp.)

2008 Cartas Mapuches. Siglo XIX, Ediciones Colibrí \& Ocho libros.

Pinto, Jorge

2002 La formación del Estado y la nación y el pueblo mapuche, DIBAM, Santiago, 2da. Ed. Revisada.
Pinto, Jorge

2015a "Los orígenes del conflicto Estado-pueblo mapuche en el siglo XX", en: Conflictos étnicos, sociales y económicos. Araucanía, 1900-2014, Pinto, Jorge (Ed.), Pehuén, Santiago, pp. 49-90.

Pinto, Jorge (Ed.)

2015b Conflictos étnicos, sociales y económicos. Araucanía, 1900-2014, Pehuén, Santiago.

Pinto, Jorge

2015c "Colonos, ocupantes nacionales, campesinos y obreros de La Araucanía, 1900-1973", en: Conflictos étnicos, sociales y económicos. Araucanía, 1900-2014, Pinto, Jorge (Ed.), Pehuén, Santiago, pp. 91-135.

Pinto, Jorge

2015d "El bandolerismo en la Frontera, 1880-1929", en: Conflictos fronterizos en la Araucanía, Ediciones Universidad de La Frontera, Temuco, pp. 105-127.

Riquelme, Patricio et al. (Comps.)

2008 Archivos locales de las grandes familias Mapuches de Malleco en el siglo XIX, sin editorial, Temuco.

Saavedra, Cornelio

1870 Documentos relativos a la ocupación de Arauco, Cámara Chilena de la Construcción, Santiago, 2008.

Torrealba Zenteno, Agustín

1907 Tierras del Estado i radicación de indígenas, Imprenta, Litografía y Encuadernación Barcelona, Santiago.

Urrutia, Basilio

1876 "Memoria del Jeneral en Jefe del Ejército del Sur", en: Memoria del Ministerio de Guerra y Marina, Imprenta de la Librería de El Mercurio, Santiago, pp. 5-10.

Varela, José Miguel

2015 Un veterano de tres guerras, Editorial Academia de Historia Militar, Santiago de Chile.

Vera, Robustiniano

1905 La pacificación de Arauco, Imprenta El Debate, Santiago. Versión digitalizada (http://www.memoriachilena.cl/602/ w3-propertyvalue-127682.html).

Villalobos, Sergio

1982 "Tres siglos y medio de vida fronteriza”, en: Relaciones fronterizas en La Araucanía, Villalobos, Sergio et al, Ediciones Universidad Católica de Chile, Santiago, pp. 9-64.

Villalobos, Sergio

1984 "Reseña de Arturo Leiva, El primer avance a la Araucanía. Angol, 1862, Universidad de La Frontera, Temuco, 1984", en: Historia $\mathrm{N}^{\circ}$ 20, Santiago, pp. 519-523.

Villalobos, Sergio

1995 Vida fronteriza en La Araucanía, Editorial Andrés Bello, Santiago.

\section{Notas}

1 Que comprende aproximadamente las regiones VIII a X de Chile, entre los ríos Biobío y Toltén.

2 En otra parte señala: "Existe una vida fronteriza con relaciones múltiples e intensas, característica de las áreas de contacto apacibles, donde el choque violento es solo esporádico" (Villalobos, 1984: 521).
"The war of every man against every man" (Leviathan, Cap. 13, 13: 85).

4 En un trabajo anterior había señalado lo contrario: "A fines del siglo XVIII, los aspectos violentos parecían perder terreno en la Araucanía histórica, ante el avance disolvente de un nuevo estilo de vida marcado por nuevos hábitos 
de consumo y de relaciones políticas" (León Solís, 1990: 225).

$5 \quad$ En las postrimerías del siglo XVIII: "El universo humano que floreció en las fronteras fue pintoresco y violento... La sociedad europea y la tribal se unían en una danza de solidaridad de marginados, embriagados en el goce de una libertad irrestricta" (León Solís, 1990: 215).

$6 \quad$ Al respecto, véase, entre otros: Bengoa (2007); Contreras Painemal (2010); León Solís (1992 y 1993) y Marimán (2006: 77-81).

7 Nagche: gente de los territorios abajinos a la cordillera de Nahuelbuta (Angol, Los Sauces, etc.).

8 Unidad territorial básica mapuche, en la actualidad se les conoce como Lof Lafkenche: gente de los territorios junto al mar (Arauco, Cañete, Tirúa, etc.).

9 Wenteche: habitantes de la zona central de la actual Región de La Araucanía (Lautaro, Collipulli, Lumaco, etc.).

10 Pewenche: gente de los territorios en la cordillera de los Andes (Alto Biobío, Lonquimay, Llaima, etc.).

11 El alzamiento coincidió con la guerra civil entre federalistas y centralistas, donde los mapuches establecieron alianzas con ambos bandos (Marimán, 2006: 102; Pinto, 2002: 144). En cambio, para Bengoa (1985: 168): "La revolución del 59 provocó un alzamiento casi general de los mapuches. Con excepción de las agrupaciones costinas del sur, muy ligadas a Valdivia, y las agrupaciones de Choll Choll y Purén (Coñoepán y Catrileo), todas las demás se sublevaron, impulsadas básicamente por dos factores: el avance en el norte de la frontera del Biobío, y por el sur, la presencia de los colonos alemanes. Los boroanos, por lo general fuera de la alianza arribana [wenteche], se integraron al levantamiento, y también lo hicieron muchos grupos abajinos [nagche] dirigidos por el cacique Domingo Melín".

12 "El arma diplomática fue utilizada por Saavedra con singular maestría, realizando numerosos parlamentos y sabiendo utilizar el engaño, el halago, y ciertamente, la división interna entre los mapuches" (Bengoa, 1985: 175).

13 Saavedra, Cornelio, "Memoria de 1868. Establecimiento de la línea del Malleco", reproducido en Saavedra (1870: 98-103).

14 Como señala Bengoa (1985: 175): “Saavedra se oponía al despojo total de los indígenas, consecuencia casi cierta del sistema de colonización espontánea, el cual, opinaba, no era bueno para nadie". En cambio, agrega, "hubo sectores militares -como José Manuel Pinto, que reemplazó a Saavedra- quienes abogaron directamente por el exterminio de los mapuches como única solución a la ocupación de la Araucanía".

15 "Con el avance de la línea de fuertes del Biobío al Malleco, en 1868, adquirió el Fisco, por medio de escrituras de compraventa, extensos terrenos baldíos, antes ocupados por indígenas, cuya mensura y enajenación se llevó a cabo en los años 1868 a 1881. Hasta este tiempo intervinieron en cuestiones y para determinar la merced de indígenas, el Protector de Indígenas, el Sr. Intendente de la Provincia y (el) Juez de Letras" (Teodoro Schmidt, Memoria de la Comisión de Ingenieros correspondiente al año de 1890, Temuco, 27 de mayo de 1891, 10 fojas sin numeración, en: Archivo Nacional, Archivo de la Intendencia de Cautín, Vol. 50 Asignaciones de terrenos, 1891-1892).
16 Teodoro Schmidt, Memoria de la Comisión de Ingenieros correspondiente al año de 1890, Temuco, 27 de mayo de 1891, 10 fojas sin numeración, en: Archivo Nacional, Archivo de la Intendencia de Cautín, Vol. 50.

17 Debe tratarse del coronel Vicente Villalón, intendente de la provincia de Arauco entre el 19 de noviembre y el 30 de diciembre de 1859 (Anuario Estadístico, 1870: 249).

18 Mangil Wenu, "Carta al Presidente de la República de Chile, Manuel Montt, Mapu, septiembre, 21 de 1860", recopilada por Jorge Pavéz (2008: 321). Publicada originalmente en: El Mercurio, Valparaíso, 13 de mayo de 1861.

19 "Domingo Salvo", (sitio web) Santa Bárbara-Bío-Bío http://santabarbarabiobio.blogspot.cl/2009/02/domingosalvo.html. Consultado el 4 de agosto de 2016. En la expedición de fines de 1859, la tropa de Domingo Salvo estaba compuesta por cerca de 400 milicianos de caballería “conocidos generalmente con el nombre de lleulles, [que] iban por su propia cuenta, sin más incentivo que el saqueo que pudieran efectuar entre los indios bien poco armados con armas de fuego, y los más con picas y laques" (Navarro, 1907: 45). Navarro sostiene que Salvo era "el hombre más versado que había en conocimientos y costumbres de los indios por la larga permanencia que tuvo entre ellos durante el período de Benavides y los Pincheiras". Citando a Horacio Lara, afirma que "era tan terrible el nombre de Salvo entre los indios que lo consideraban un ser extraño y supersticioso", siendo capaz de infundir temor ante la sola mención de su nombre (Navarro, 1907: 39 y 32).

20 Nidol Toki: comandante principal de las fuerzas militares mapuches.

21 Weichan: Ir a la guerra; Mapu: Tierra/Territorio. Weichanmapu: Tierras/Territorios en guerra.

22 Denominación hispano-chilena para referirse a los mapuches.

23 Carta del intendente de Valdivia, Epifanio del Canto, al ministro de Guerra, Francisco Echaurren, 20 de mayo de 1871, en: Archivo Nacional, Ministerio de Guerra, Vol. 597, sin fojas.

24 El Meteoro, Los Ángeles, 12 de enero de 1869, cit. por Bengoa (1985: 217).

25 "Podemos decir que la guerra de ocupación de la Araucanía fue...una guerra regular en la forma, pero una guerra completamente irregular en el despliegue efectivo" (Ancán, 2008: 17).

26 "Sumario indagatorio seguido a los indios muertos el 12 del presente mes en el calabozo de este cuartel", Lumaco, noviembre 11 (sic) de 1881, en: Riquelme et al (2008: 136-200).

27 Carta de M. Antonio Neculmán, presidente de la Sociedad Caupolicán, al intendente de la provincia de Cautín, Temuco, 23 de julio de 1913, sin fojas, en: Archivo Nacional, Intendencia de Cautín, Vol. 111 (comunicaciones recibidas, 1911-1916). Se ha modernizado la ortografía para facilitar la lectura.

28 Carta de M. Antonio Neculmán, al intendente de la provincia de Cautín, Temuco, 23 de julio de 1913, sin fojas, en: Archivo Nacional, Intendencia de Cautín, Vol. 111, sin fojas.

29 Carta de M. Antonio Neculmán intendente de la provincia de Cautín, Temuco, 23 de julio de 1913, sin fojas, en: Archivo Nacional, Intendencia de Cautín, Vol. 111, sin fojas. 
Carta de M. Antonio Neculmán intendente de la provincia de Cautín, Temuco, 23 de julio de 1913, sin fojas, en: Archivo Nacional, Intendencia de Cautín, Vol. 111, sin fojas.

31 Carta de M. Antonio Neculmán intendente de la provincia de Cautín, Temuco, 23 de julio de 1913, sin fojas, en: Archivo Nacional, Intendencia de Cautín, Vol. 111, sin fojas.

32 Leoncio Rivera, Informe Anual de la Comisión Radicadora de Indígenas, Temuco, Abril de 1912, en: Archivo Nacional, Fondo Intendencia de Cautín, Vol. 115 (Comunicaciones recibidas, 1912-1914), Fojas 1-8. Se ha modernizado la ortografía para facilitar la lectura.

33 Esteban Romero, Carta al Presidente de la República, Chillán, 10 de noviembre de 1896, en: Pavéz (805-815).

34 "Las tierras de Arauco". Presentación de la Sociedad Caupolicán al inspector general de colonización e inmigración, 1915, en: La Época, 23 de octubre de 1915, Temuco, reproducido como apéndice en: Pinto (2015b: 286-288).
35 La descripción más completa de los métodos de expoliación empleados en Valdivia y Llanquihue contra la población mapuche regional la proporciona Torrealba (1907), que excluye expresamente los medios "más inhumanos y criminales que consisten en hacer desparecer al indígena comunero" (Cit. por Foerster, 1996: 294).

36 Acerca de Joaquín Mera, a quien el padre Sigisfredo califica de "tristemente célebre como usurpador, incendiario y asesino" (Fraunhäusl, 1904-1924: 299) véase, del mismo autor: (1904-1924: 306-309, 313 y 344-345) y Díaz Mesa (1907: 217; 239-240; 245-246; 250-252 y 258-260).

37 Archivo Nacional, Intendencia de Cautín, Vol. 111, sin fojas.

38 Véase: Bengoa (1985: 380-381); Pinto (2015a: 68-71) y la documentación reunida por Pinto (2015c: 300-315).

39 Recuérdese la matanza de Suto, en Loncoche, actual provincia de Cautín, en 1910 (Berger, 2014; Pinto, 2015c: 94-101 y la documentación de prensa recogida por el mismo autor, Pinto, 2015b: 288-299). 
